\title{
Conocimientos y estado de salud bucal en madres beneficiarias del sistema Chile Crece Contigo
}

\author{
RICARDO CARTES-VELÁSQUEZ ${ }^{(1)}$, SANDRA MARDONES ${ }^{(2)}$ y CONSTANZA PAREDES ${ }^{(2)}$
}

\section{RESUMEN}

Su objetivo es realizar un diagnóstico del estado de salud bucal y de conocimientos de las madres beneficiarias del sistema Chile Crece Contigo en la comuna de Alto Bío Bío y sus posibles relaciones con variables sociodemográficas. Se trata de un estudio analítico de corte transversal, mediante un muestreo estratificado por conglomerados se obtuvo una muestra de 70 madres. Se realizó un examen bucal bajo los criterios de la OMS y se aplicó un cuestionario de 23 preguntas en formato de entrevista guiada, en relación a medidas de autocuidado, enfermedades más prevalentes y anatomía bucodentaria. Los resultados muestran un COPD de 14,6 $\pm 5,52$ siendo mayor en multíparas y mujeres mayores de 20 años. El IHO-S alcanzó un valor de 0,83 $\pm 0,54$, más alto en multiparas. El IPC fue mayoritariamente de nivel 2. Con respecto al conocimiento de salud bucal este alcanzó un valor de 13,03 $\pm 2,70$, siendo mayor en multíparas.: La población estudiada muestra un nivel muy alto de daño bucal y limitados conocimientos de salud oral. Esto empeora en la población pehuenche y con menor instrucción. Las mujeres multíparas y de mayor edad empiezan a acceder mayoritariamente a tratamientos de urgencias que llevan a la pérdida de sus dientes. Dada la evidencia científica y los resultados del presente estudio, la inclusión del componente salud bucal en el ChCC aparece como una medida costo-efectiva para mejorar en el mediano y largo plazo el actual panorama.

Palabras clave: salud pública, odontología, sistema, embarazada, madre, niño.

\section{ABSTRACT}

\section{KNOWLEDGE AND ORAL HEALTH STATUS IN MOTHERS WHO RECEIVE BENEFITS FROM THE CHILE CRECE CONTIGO (CHILE GROWS WITH YOU) PROGRAM}

The objective of this study was to assess oral health status and knowledge of mothers that receive benefits from the "Chile Grows With You" program in the Alto Bio Bio county, and possible relationships with sociodemographic variables. This was a cross-sectional, analytical study. Cluster sampling was used to obtain a sample of 70 mothers. In order to obtain information on self care, prevalent diseases, and oral and dental anatomy, each mother was given an oral exam, preformed according to WHO criteria, and a 23 item questionnaire, applied in a guided interview format. The results show a DMFT of $14.6 \pm 5.52$, which was higher in multiparous women and in women older than 20. The OHI-S was $0.83 \pm 0.54$, higher in multiparous women. The CPITN was mainly level 2. For oral health knowledge, there was an average score of $13.03 \pm 2.70$, higher in multiparous women. The studied population shows a very high level of oral damage, and limited oral health knowledge. This was worse in Pehuenche populations and among those with less education. Multiparous and older women mainly access only emergency treatment, which leads

(1) Cirujano Dentista. Etapa de Destinación y Formación. CESFAM Ralco Alto Bío-Bío. Servicio de Salud Bío-Bío. Villa Ralco s/n. Alto Bío-Bío. VIII Región. Chile. cartesvelasquez@gmail.com.

(2) Interna de Odontología. Universidad de Concepción. VIII Región. Chile. 
to the loss of teeth. Given the scientific evidence and the results of the present study, the inclusion of oral health in the "Chile Grows With You" program appears to be a cost effective measure to improve this situation in the medium and long term.

Keywords: public health, dentistry, system, pregnancy, mother, child.

\section{INTRODUCCIÓN}

La implementación del sistema Chile Crece Contigo (ChCC) es probablemente la política pública de bienestar social de mayor importancia de la última década en nuestro país. En ella la participación de una serie de instituciones tiene como objetivo velar por la adecuada protección social de los ciudadanos y desarrollo de las personas, poniendo especial énfasis en la primera infancia, etapa clave en la formación del ser humano.

Las instituciones sanitarias, dado lo anterior, han tomado un papel preponderante en la ejecución del ChCC, asumiendo como grupo inicial y prioritario de atención a todas las embarazadas del país, las que acceden a una serie de prestaciones entre las que destacan atenciones médicas, beneficios sociales, actividades de educación en temas promocionales y preventivos, entre otras. Sin embargo, no existe una mención explícita del componente de salud bucal, ni tampoco de intervenciones a desarrollar en este grupo al respecto.

La literatura nos muestra una amplia evidencia acerca de la interrelación existente entre la salud general y bucal de las personas, hecho aún más notorio en el grupo de las gestantes ${ }^{1,2}$, así como también del positivo efecto que tienen las intervenciones recuperativas, promocionales y preventivas en la salud bucal de la embarazada $\mathrm{y}$, posteriormente, en el binomio madre-hijo $\mathrm{o}^{3-13}$. Esto debido al carácter infecto-contagioso de las enfermedades bucales ${ }^{14,18}$. En este sentido, la salud oral debiese también tener un espacio oficial en el sistema ChCC, más allá de que en forma complementaria se desarrollen otras estrategias como la reciente implementación del piloto del GES odontológico en la embarazada o la existencia de metas asociadas en los Indicadores de Atención Primaria de Salud (IIAPS) o de Desempeño Colectivo enfocadas en este grupo.
A pesar de lo anterior, no es menos cierto que la existencia de adecuados diagnósticos es el primer e imprescindible paso para llegar a la implementación de políticas efectivas, idealmente costo-efectivas. De esta manera, el presente trabajo tiene como objetivo determinar el estado de salud bucal y los conocimientos que tienen en esta materia las madres que son beneficiarias del sistema $\mathrm{ChCC}$ en la comuna de Alto Bío-Bío, así como encontrar las posibles relaciones con variables sociodemográficas. Lamentablemente no contamos con diagnósticos locales previos que nos puedan aportar datos epidemiológicos de base, por lo que utilizaremos como referencia un estudio hecho en primigestas de la aledaña comuna de Santa Bárbara el año 2006, el que arrojó una prevalencia de $75 \%$ de caries en ese grupo ${ }^{19}$.

Por último, debemos destacar que nuestra comuna está caracterizada por una alta ruralidad y presencia de población indígena pehuenche, fue elegida como una de las 161 comunas pilotos donde se implementó el sistema $\mathrm{ChCC}$ en julio del 2007, y está considerada además como comuna vulnerable.

\section{MATERIAL Y MÉTODO}

Se trata de un estudio analítico de corte transversal, en el período abril-julio del año 2009, en madres beneficiarias del sistema ChCC, de la comuna de Alto Bío-Bío, cuyo parto hubiese sido entre julio de 2007 y diciembre de 2008.

La selección de la muestra se hizo mediante un muestreo estratificado por conglomerados, en función de las 9 comunidades o sectores que conforman la comuna. Lamentablemente 2 comunidades no pudieron ser visitadas debido a situaciones de violencia en ellas al momento de la recolección de los datos. De esta manera, el universo del estudio fue de 74 mujeres repartidas en 7 comunidades. 
Tabla 1. Caracterización de las madres beneficiarias del sistema ChCC, Alto Bío Bío.

\begin{tabular}{|c|c|c|c|c|}
\hline \multirow{2}{*}{ Edad } & $<20$ años & $\begin{array}{c}\text { Primi- } \\
\text { gestas }\end{array}$ & Multíparas & Total \\
\cline { 2 - 5 } & $>20$ años & 13 & 0 & 16 \\
\hline \multirow{2}{*}{ Instrucción } & $\begin{array}{c}\text { Media } \\
\text { incompleta }\end{array}$ & 21 & 34 & 2 \\
\cline { 2 - 5 } & $\begin{array}{c}\text { Media } \\
\text { completa }\end{array}$ & 8 & 7 & 15 \\
\hline \multirow{3}{*}{ Etnia } & Occidental & 6 & 4 & 10 \\
\cline { 2 - 5 } & Pehuenche & 23 & 37 & 60 \\
\cline { 2 - 5 } & Total & 29 & 41 & 70 \\
\hline
\end{tabular}

El tamaño de la muestra se definió según la fórmula de estimación de muestras para una población finita. Los valores para $\mathrm{z}$ y d fueron de $95 \%$ y $5 \%$, respectivamente. Los de p y q se corresponden a los encontrados en Santa Bárbara el 2006 , esto es $75 \%$ y $25 \%$ respectivamente, lo que determinó una muestra inicial de 59 mujeres. Pero dada la imposibilidad de cubrir 2 comunidades, se amplió proporcionalmente la muestra en las 7 restantes hasta alcanzar una muestra final de 70 mujeres, lo que significa una precisión absoluta del 2,4\% para un nivel de confianza del $95 \%$.

Los datos fueron recolectados a través de visitas domiciliarias a las mujeres de las diferentes comunidades, tras haber explicado previamente a las pacientes de que se trataba el estudio y pedirles su consentimiento para examinarlas. Dicha recolección de información se realizó en una habitación tranquila, con un observador único y con un asistente que registró la información en una ficha adecuada para el estudio. El examen clínico se hizo mediante inspección visual bajo luz artificial, utilizando para ello una sonda curva de caries y un espejo \#5. Para evaluar la presencia de enfermedad gingival se secó la encía con gasa observando de esta forma la apariencia que presenta, además de realizar el debido examen periodontal con una sonda periodontal de la OMS para la determinación del Índice Periodontal Comunitario (IPC). Para medir la historia de caries se usó el índice COPD de Klein y Palmer para piezas permanentes. Los criterios para el diagnóstico del estado de salud dentaria utilizados en este estudio son los descritos por la OMS.

El nivel de conocimientos en salud bucal se determinó en base a un cuestionario de 23 preguntas que cubrían aspectos del cuidado de salud bucal, enfermedades prevalentes y anatomía bucodentaria básica. Este cuestionario fue aplicado previamente en otro estudio en embarazadas realizado por una de las autoras, y en el presente estudio se aplicó en formato de entrevista guiada en razón del analfabetismo de una parte del grupo evaluado.

La tabulación de los datos y la representación de los gráficos y tablas se realizó en el software MS Excel 2003.

Para el análisis estadístico se usó el test de

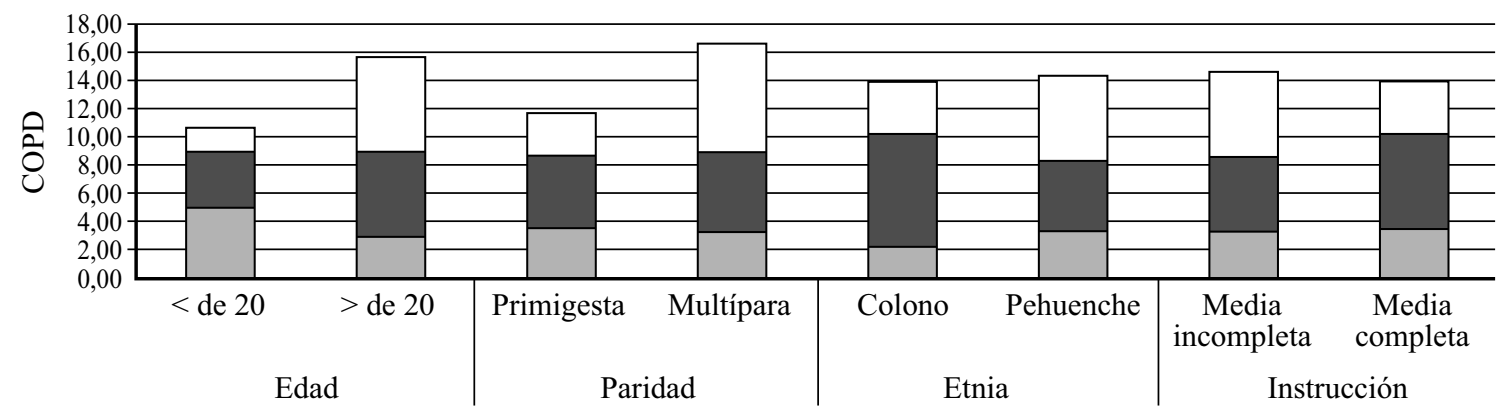

Variables sociodemográficas

\begin{tabular}{l}
$\square(\mathrm{COPD}) \quad \square \mathrm{O}(\mathrm{COPD}) \quad \square \mathrm{P}(\mathrm{COPD})$ \\
\hline
\end{tabular}

Figura 1. Promedio de COPD según edad, paridad, etnia y grado de instrucción. 


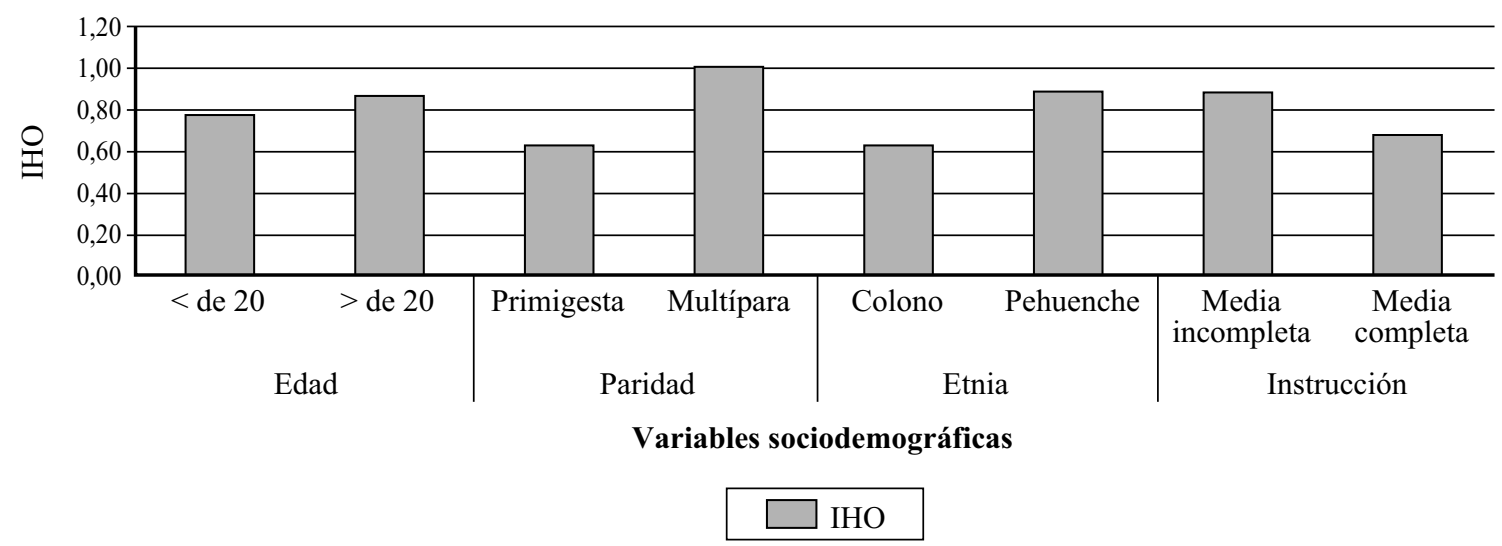

Figura 2. Promedio de IHO-S según edad, paridad, etnia y grado de instrucción.

student con un nivel de significación del 5\% con el software Stata/SE 10.

\section{RESULTADOS}

En la Tabla 1 podemos apreciar la caracterización de la población en estudio, la que corresponde principalmente a mujeres mayores de 20 años $(27,28 \pm 7,3)$, con educación básica completa $(75,7 \%)$, de etnia pehuenche $(85,7 \%)$ y con más de 1 hijo $(58,6 \%)$.

La historia de caries (prevalencia) fue de un $100 \%$ para la población estudiada. En el daño por caries, medido por el índice COPD (Figura 1 ), vemos que el promedio general fue de 14,6 $\pm 5,52$, alcanzando mayores valores en mujeres mayores de 20 años $(15,76 \pm 5,2)$, multíparas $(16,66 \pm 5,0)$, pehuenches $(14,7 \pm 5,64)$ y en las mujeres con educación media incompleta $(14,76$ $\pm 5,63)$. Las diferencias fueron estadísticamente significativas en relación a la edad $(\mathrm{p}=0,0009)$ y a la paridad $(\mathrm{p}=0,0001)$.

La higiene bucal, valorada a través del IHO-S (Figura 2) tuvo un promedio de $0,83 \pm 0,54$, donde los grupos de mujeres con peor higiene fueron las mayores de 20 años $(0,85 \pm 0,54)$, multíparas $(0,99 \pm 0,54)$, pehuenches $(0,87 \pm 0,56)$ y las que no habían completado la educación media $(0,87 \pm 0,57)$, dichas diferencias fueron estadísticamente significativas sólo en paridad $(\mathrm{p}=0,0041)$.

El estado de salud periodontal, medido por el IPC, en 51 (72,9\%) de las pacientes alcanzó un valor de 2 , siendo el más frecuente, no encontrándose diferencias estadísticamente significativas en ningunas de las variables estudiadas.

Finalmente en la evaluación de los conocimientos de salud bucal por medio del cuestionario (Figura 3), se encontró un promedio general de 13,03 $\pm 2,70$; valores superiores se encontraron en los grupos de las mujeres mayores de 20 años $(13,28 \pm 2,76)$; en las multíparas $(13,59 \pm 2,77)$; en las occidentales $(13,5 \pm 2,32)$, y en las con educación media completa $(14,2 \pm 2,04)$. Estas diferencias sólo fueron estadísticamente significativas en la paridad $(\mathrm{p}=0,0391)$.

\section{DISCUSIÓN}

Las políticas en salud bucal se han centrado básicamente en los niños, comenzando con los de 6 y 12 años, extendiéndose luego a la población menor de 20 años y a las embarazadas. En este último grupo no encontramos diagnósticos nacionales, como sí ocurre en los de 12 años ${ }^{20}$, por lo que en virtud de este nuevo sistema ChCC se hace necesario contar con uno que permita conocer con exactitud el estado de salud bucal de las embarazadas y, además, el nivel de conocimientos que tienen al respecto. Ello porque existe una clara evidencia de que esto será un factor determinante en la salud de 
sus hijos y la propia. Así, con datos duros, podemos empezar a planificar e implementar las estrategias necesarias para el empoderamiento de las personas en su propia salud, objetivo clave en el nuevo modelo de salud familiar.

El estudio arrojó resultados bastante alarmantes, pero que lamentablemente se condicen con las estadísticas y tendencias presentes en la población rural de nuestro país, que persiste como la gran deuda del sistema sanitario chileno ${ }^{21}$. Respecto de la historia de caries, el $100 \%$ supera todos los valores encontrados en poblaciones similares ${ }^{22,23}$ y en algunas cercanas a nuestra comuna ${ }^{19}$. $\mathrm{Si}$ revisamos el daño por caries, COPD de 14,6, vemos que también superan a dichos estudios. Además, como era de esperar el daño aumenta con la paridad y por consiguiente con la edad, pero en un análisis más fino podemos ver que en estas dos variables el aumento es a expensas del componente $\mathrm{P}$, vale decir que las mujeres multíparas y/o mayores de 20 años básicamente comienzan a tener un acceso a tratamiento dental de urgencia que, finalmente, determina un mutilamiento de su sistema estomatognático. Un comportamiento similar vemos al analizar las variables etnia e instrucción donde las mujeres pehuenches y con educación media incompleta, si bien tienen un COPD similar a sus contrapartes occidentales y con mayor instrucción, en su caso es debido a una mayor proporción de dientes perdidos. Lo anterior hace necesario, en primer lugar, un constante mejoramiento y aumento de las actividades promocionales y preventivas; en segundo, un aumento en las coberturas y posibilidades de acceso a tratamiento odontológico y, en tercero, que éste sea oportuno, para que el daño por caries sea menor o en su defecto sea mayoritariamente daño-tratado y no daño-activomutilante como existe en este momento.

Cuando vemos la higiene oral se da una relación de variables prácticamente igual al daño por caries. Esto refuerza la idea de aumentar y mejorar las actividades promocionales, no tan sólo en virtud de la salud de la madre sino del binomio que hace con cada uno de sus hijos. Ello porque como ha sido demostrada la actitud de los padres es uno de los factores más determinantes en las conductas de sus hijos en materia de cuidado de la salud bucal ${ }^{6,9,12,13}$. El estado de salud periodontal no hace más que volver a confirmar los planteamientos anteriores.

El estudio además de valorar el estado de salud de las madres, se orientó a determinar cuál era el nivel de conocimiento respecto de

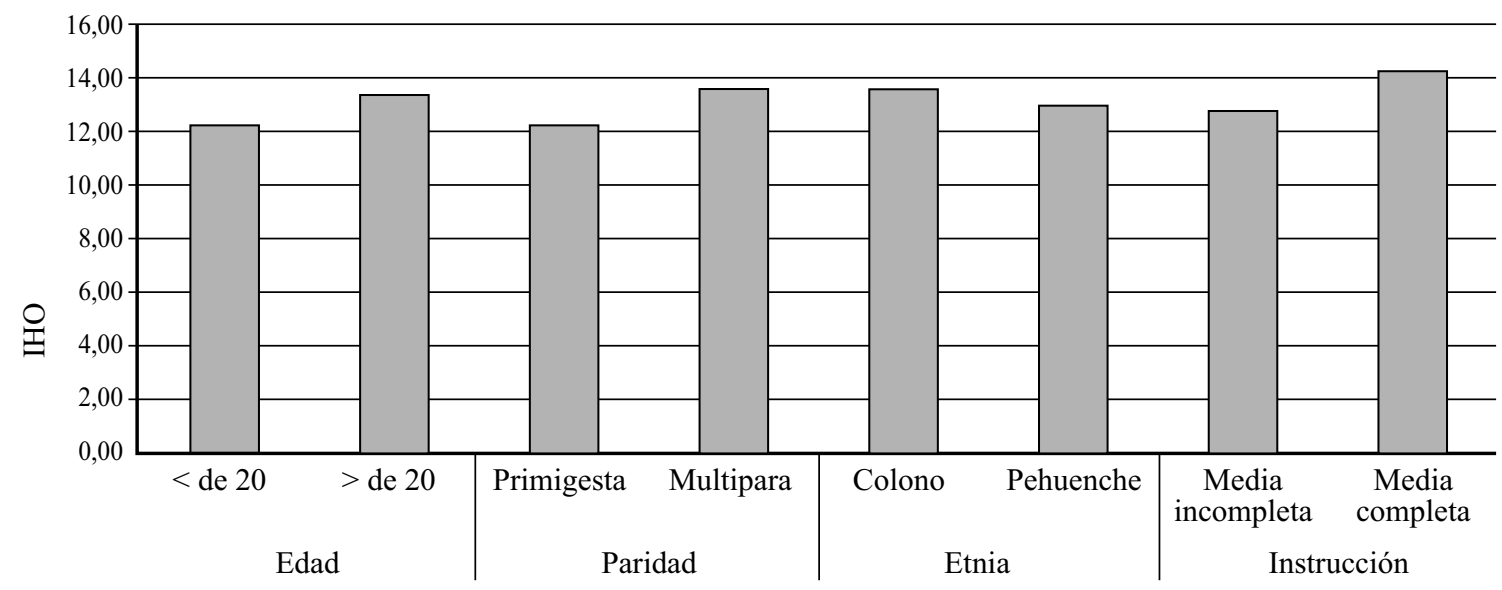

Variables sociodemográficas

Puntaje

Figura 3. Promedio de puntaje del cuestionario de conocimientos en salud bucal según edad, paridad, etnia y grado de instrucción. 
la salud bucal en general. Así, los resultados en términos comparativos al interior de los grupos fueron esperables, con puntajes más alto para las mujeres con mayor instrucción, edad y occidentales en línea con los resultados anteriores. Pero también nos muestra, en general, pobres conocimientos que promediaron un 56\% de respuestas correctas, resultados que también encontramos en poblaciones similares y que se relacionan con diversos mitos y creencias acerca de la salud bucal de las embarazadas ${ }^{22,24}$, factores muy importantes que no sólo determinan comportamientos acerca de la búsqueda de prestaciones de salud para si mismas ${ }^{25,26}$ sino que lo harán en un futuro para las prestaciones que soliciten para sus hijos ${ }^{6}$; además de la falta de medidas de autocuidado, lo que mantiene el círculo vicioso de un alto daño bucal representado por una mayor proporción de dañoactivo-mutilante, tal como lo vemos en esta población.

La existencia del sistema ChCC brinda la oportunidad de albergar y garantizar una serie de prestaciones que resguarden la calidad de vida de los futuros ciudadanos y la salud bucal es un importante factor de la calidad de vida, hecho aún más notorio en los grupos desfavorecidos, por lo que un lugar al interior de este sistema se muestra como una situación totalmente posible e incluso necesaria.

A la luz de estos resultados y de la evidencia, la implementación explícita y oficial de actividades asistenciales, pero básicamente de promoción y prevención en el ChCC, permitiría de manera costo-efectiva, en virtud del modelo de salud familiar y empoderando a la población, mejorar en el mediano y largo plazo la actual situación de alto daño que muestra la población de madres beneficiarias de este sistema y a futuro la de sus hijas e hijos, principales objetivos del mismo.

\section{REFERENCIAS}

1. ACHARYA S, BHAT P. Oral-Health-Related Quality of Life during Pregnancy. J Public Health Dent. 2009;69(2):74-77.

2. LAINE MA. Effect of pregnancy on periodontal and dental health. Acta Odontol Scand 2002; 60: 257 -264.
3. GOMEZ S, WEBER A. Effectiveness of a caries preventive program in pregnant women and new mothers on their offspring. Int J Paediatric Dent 2001; 11: 117-122.

4. COGULU D, KOCATAS N, UZEL A, ERONAT N, AKSIT S. A long-term effect of caries-related factors in initially caries-free children. Int J Paediatr Dent 2008; 18: 361- 367.

5. GUNAY H, DMOCH-BOCKHORN K, GUNAY Y, GEURTSEN W. Effect on caries experience of a longterm preventive program for mothers and children starting during pregnancy. Clin Oral Invest 1998; 2: 137-142.

6. WATOSN M-R, HOROWITZ AM, GARCIA I, CANTO MT. Caries conditions among 2-5-year-old immigrant Latino children related to parents' oral health knowledge, opinions and practices. Community Dent Oral Epidemiol 1999; 27: 8-15.

7. KAWASHITA Y, FUKUDA H, KAWASAKI K et al. Dental Caries in 3-Year-Old Children is Associated More with Child-Rearing Behaviors than MotherRelated Health Behaviors. J Public Health Dent. 2009; 69(2): 104-110.

8. PLUTZER K, SPENCER AJ. Efficacy of an oral health promotion intervention in the prevention of early childhood caries. Community Dent Oral Epidemiol 2008; 36: 335-346.

9. OKADA M, KAWAMURA M, KAIHARA Y et al. Influence of parents' oral health behaviour on oral health status of their school children: an exploratory study employing a causal modelling technique. Int Journal Paediatric Dent 2002; 12: 101-108.

10. CANÇADO M, LOPEZ M. La clínica odontológica del bebé integrando un servicio de salud. Arch Pediatr Urug 2008; 79(2): 150-156.

11. GREMBOWSKI D, SPIEKERMAN C, MILGROM $P$. Linking mother access to dental care and child oral health. Community Dent Oral Epidemiol 2009; 37 : 381-390.

12. MOHEBBI S, VIRTANEN J, MURTOMAA H, VAHID-GOLPAYEGANI M, VEHKALAHTI M. Mothers as facilitators of oral hygiene in early childhood. Int J Paediatric Dent 2008; 18: 48-55.

13. OKADA M, KAWAMURA M, HAYASHI Y, TAKASE N, KOSAI K. Simultaneous interrelationship between the oral health behavior and oral health status of mothers and their children. J Oral Sci 2008; 50: 447-452.

14. KOBAYASHI N, ISHIHARA K, SUGIHARA N, KUSUMOTO M, YAKUSHIJI M, OKUDA K. Colonization pattern of periodontal bacteria in Japanese children and their mothers. J Periodont Res 2008; 43: 156-161.

15. HERRERA C, PANTOJA P, DE LA MAZA T, SANHUEZA A, SALAZAR L. Diagnóstico microbiológico y molecular de bacterias cariogénicas en mujeres embarazadas de la Región de La Araucanía, Chile. Rev Chil Infect 2007; 24 (4): 270-275.

16. YANG EY, TANNER ACR, MILGROM P et al. 
Periodontal pathogen detection in gingiva/tooth and tongue flora samples from 18- to 48-month-old children and periodontal status of their mothers. Oral Microbiol Immunol 2002: 17: 55-59.

17. THORILD I, LINDAU-JONSON B, TWETMAN S. Prevalence of salivary streptococcus mutans in mothers and in their preschool children. Int J Paediatric Dent 2002; 12: 2-7

18. PALOMER L. Caries dental en el niño. Una enfermedad contagiosa . Rev Chil Pediatr 77 (1); 5660, 2006

19. RETAMAL A, VILLALOBOS F. Estado de la salud bucal de primigestas que asisten a control prenatal al Hospital de Santa Bárbara, durante los meses de abril a junio. 2006. Pág. 60-91.

20. SOTO L, TAPIA R, JARA G, RODRÍGUEZ G, URBINA T. Diagnóstico nacional de salud bucal del adolescente de 12 años y evaluación del grado de cumplimiento de los objetivos sanitarios de salud bucal 2000-2010. Facultad de Odontología, Universidad Mayor, 2007.

21. PONTIFICIA UNVERSIDAD CATÓLICA. Encuesta nacional de salud Chile 2003. 2003. pp. 263 - 277.
22. GARBERO I, DELGADO A, BENITO DE CÁRDENAS I. Salud oral en embarazadas: conocimientos y actitudes. Acta Odontol Venez 2005; 43(3): $135-140$

23. GONZÁLEZ W, CORONA M, PINEDA A, LAO N, PARDO M. Conocimientos de las embarazadas del área de salud "Carlos J. Finlay" sobre salud bucal. Rev Cubana Estomatol 2007; 44(4): 1-5

24. FUENTES R, OPORTO G, ALARCÓN AM, BUSTOS L, PRIETO R, RICO H. Opiniones y creencias de embarazadas en control prenatal relacionadas con salud oral y embarazo. Av. Odontoestomatol 2009; 25 (3): 147-154.

25. LE M, RIEDY C, WEINSTEIN P, MILGROM P. Barriers to utilization of dental services during pregnancy: A qualitative analysis. J Dent Child 2009; 76: 46-52.

26. KUTHY R, ODOM J, SALSBERRY P, NICKEL J, POLIVKA B. Dental Utilization by Low-income Mothers. J Public Health Dent 1998;58(1):44-50.

Recepción : 19 de noviembre de 2009 Aprobación : 8 de abril de 2010

Usted puede comentar éste y otros artículos publicados en la Revista Chilena de Salud Pública, enviando un correo electrónico a revistasp@med.uchile.cl 